\title{
Characteristics of pediatric SARS-CoV-2 infection and potential evidence for persistent fecal viral shedding
}

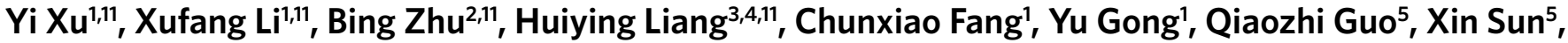 \\ Danyang Zhao5 , Jun Shen ${ }^{1}$, Huayan Zhang ${ }^{13,4,6}$, Hongsheng Liu7, Huimin Xia ${ }^{3,4,8 凶}$, Jinling Tang ${ }^{3,4} \bowtie$, \\ Kang Zhang $\mathbb{1}^{8,9,10 \bowtie}$ and Sitang Gong ${ }^{1,4} \bowtie$
}

\begin{abstract}
We report epidemiological and clinical investigations on ten pediatric SARS-CoV-2 infection cases confirmed by realtime reverse transcription PCR assay of SARS-CoV-2 RNA. Symptoms in these cases were nonspecific and no children required respiratory support or intensive care. Chest $X$-rays lacked definite signs of pneumonia, a defining feature of the infection in adult cases. Notably, eight children persistently tested positive on rectal swabs even after nasopharyngeal testing was negative, raising the possibility of fecal-oral transmission.
\end{abstract}

The outbreak of SARS-CoV-2 (formerly 2019-nCoV) infection emerged in December 2019 in Wuhan, Hubei Province, China ${ }^{1,2}$. By 25 February 2020, there had been 77,780 confirmed cases including 2,666 deaths in China and over 2,459 confirmed cases in 33 other countries $^{3}$.

The genome of the new virus and early epidemiological and clinical features of the infection in adults have been reported ${ }^{4-6}$. The infection is estimated to have a mean incubation period of $5.2 \mathrm{~d}$ and commonly causes fever, cough, myalgia and pneumonia in patients ${ }^{4}$. To date there is a paucity of information regarding SARSCoV-2 infection in children.

We here report the epidemiological and clinical features of ten children infected with SARS-CoV-2 and tested for evidence of viral excretion through the gastrointestinal and respiratory tracts.

By 20 February 2020, a total of 745 children and 3,174 adults, most of whom had either close contact with diagnosed patients or had members of the family reporting familial outbreaks in the previous 2 weeks, were screened by nasopharyngeal swab real-time PCR with reverse transcription (RT-PCR) for SARS-CoV-2 infection. Overall, 10 children (1.3\%) and 111 adults (3.5\%) tested positive. The 2.7-fold difference between children and adults is statistically significant $(P=0.002)$. All ten pediatric patients were admitted to our hospital, a treatment center for SARS-CoV-2 infection designated by the local municipal government.
Of the ten patients, six were male and four were female, with ages ranging from 2 months to 15 years (Table 1). Four had definite contact history with a confirmed patient, seven were from families with a cluster of infection and seven had travel history to epidemic areas in Hubei Province 2 weeks before the onset of infection.

Upon admission, seven had fever but none had a temperature above $39^{\circ} \mathrm{C}$ (Table 1). Other symptom presentations included coughing (five children), sore throat (four children), nasal congestion and rhinorrhea (two children) and diarrhea (three children). One child was completely asymptomatic (patient 4 ). None of the patients had other symptoms commonly seen in adult patients such as lethargy, dyspnea, muscle ache, headache, nausea and vomiting and disorientation. In fact, none of them sought medical care; they were all identified and diagnosed because of their exposure history.

Chest X-rays of these patients were either normal or showed only coarse lung markings without unilateral or bilateral pneumonia. Chest computed tomography (CT) scans showed isolated or multiple patchy ground-glass opacities in five patients but were within normal ranges in the other five. These changes were mainly seen in the outer lung fields and few patients had subpleural bands or strips. There was no pleural effusion, enlarged lymph nodes or other changes that are typically seen in the critically ill adult patients $s^{4,6}$ (Supplementary Fig. 1).

Complete blood count, urine and stool analyses, coagulation function, blood biochemistry and infection biomarkers were tested upon admission (Table 1). Almost all test results were normal in the patients except for patient 9. Few cases had leukopenia, leukocytosis, lymphopenia or elevated transaminase, which in contrast are frequently seen in adult patients. Viral testing for influenza-A virus (H1N1, H3N2, H7N9), influenza B virus, respiratory syncytial virus, parainfluenza virus, adenovirus, SARS-CoV and MERS-CoV were negative in all patients.

Out of nine patients (the tenth patient was transferred from another hospital that did not conduct cytokine tests upon admis-

\footnotetext{
'Department of Pediatric, Guangzhou Women and Children's Medical Center, Guangzhou Medical University, Guangzhou, China. ${ }^{2}$ Department of Center Laboratory, Guangzhou Women and Children's Medical Center, Guangzhou Medical University, Guangzhou, China. ${ }^{3} \mathrm{Clinical}$ Data Center, Guangzhou Women and Children's Medical Center, Guangzhou Medical University, Guangzhou, China. ${ }^{4}$ Guangdong Provincial Children's Medical Research Center, Guangzhou Women and Children's Medical Center, Guangzhou Medical University, Guangzhou, China. ${ }^{5}$ Department of Medical Administration, Guangzhou Women and Children's Medical Center, Guangzhou Medical University, Guangzhou, China. ${ }^{6}$ Division of Neonatology and Department of Pediatrics, Children's Hospital of Philadelphia and University of Pennsylvania, Philadelphia, PA, USA. ${ }^{7}$ Department of Radiology, Guangzhou Women and Children's Medical Center, Guangzhou Medical University, Guangzhou, China. ${ }^{8}$ Guangdong Provincial Key Laboratory of Research in Structural Birth Defect Disease, Guangzhou Women and Children's Medical Center, Guangzhou Medical University, Guangzhou, China. 'Guangzhou Regenerative Medicine and Health Guangdong Laboratory, Guangzhou, China. ${ }^{10}$ Center for Biomedicine and Innovations, Faculty of Medicine, Macau University of Science and Technology, Macau, China. "These authors contributed equally: Yi Xu, Xufang Li, Bing Zhu, Huiying Liang. ${ }^{凶}$ e-mail: huiminxia@hotmail.com; jltang@cuhk.edu.hk; kang.zhang@gmail.com; sitangg@126.com
} 


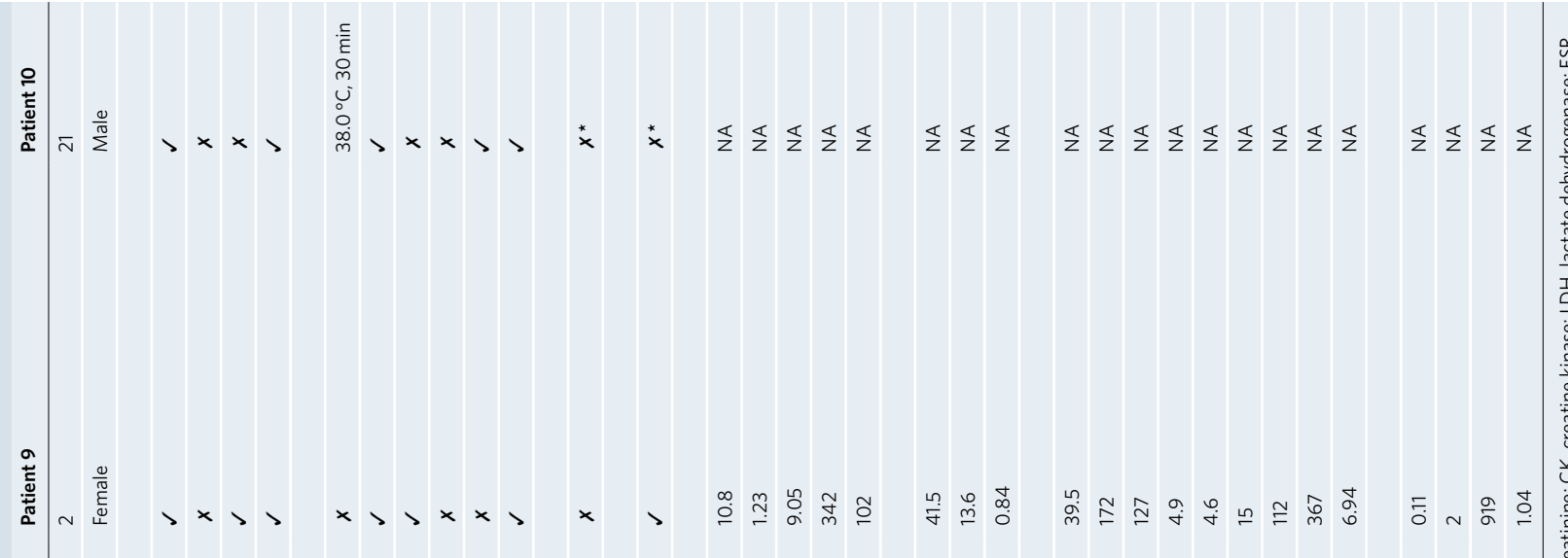

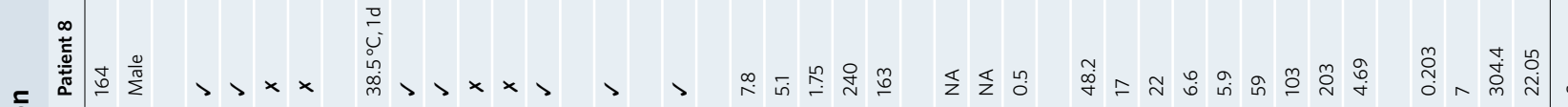

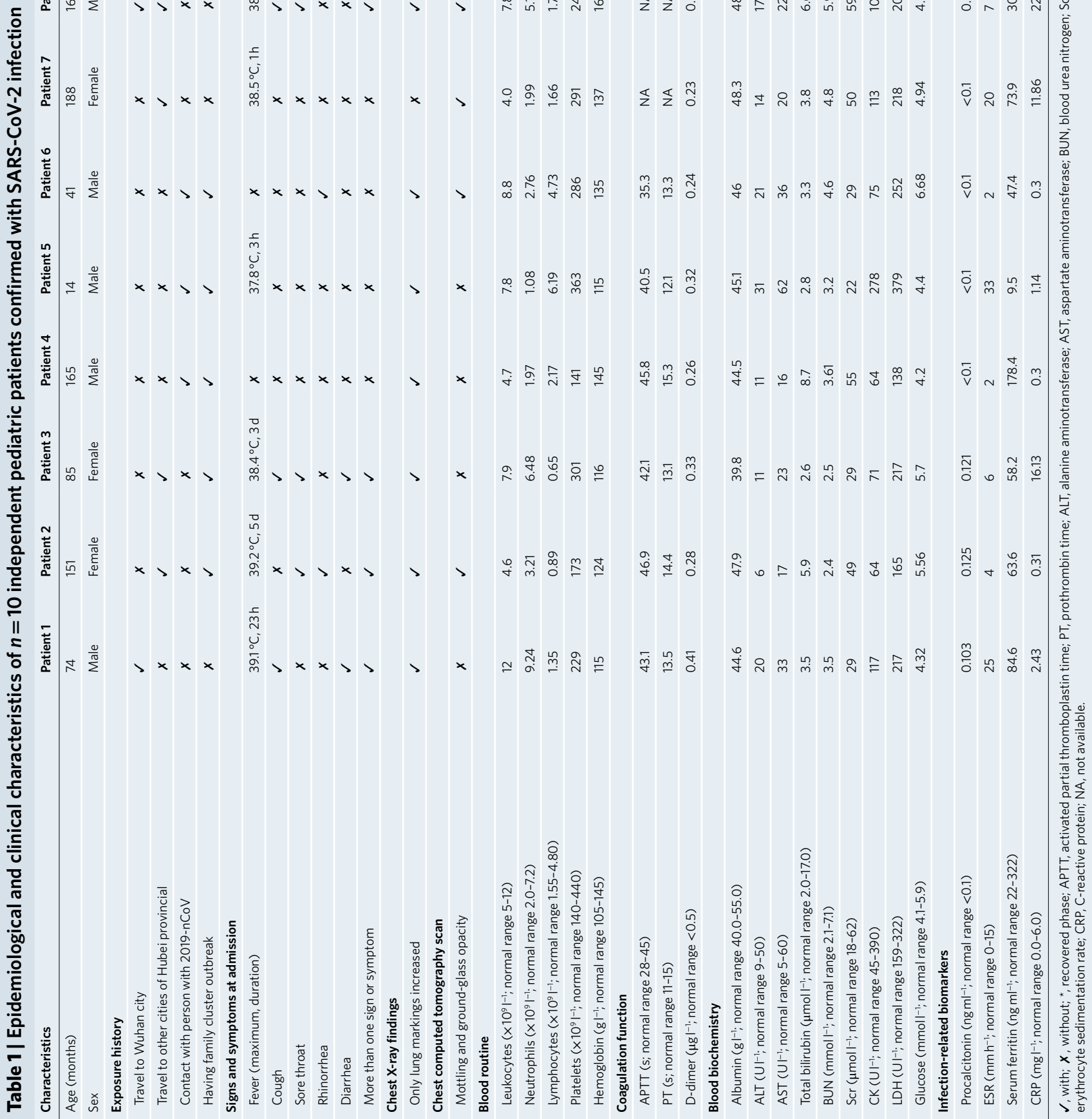




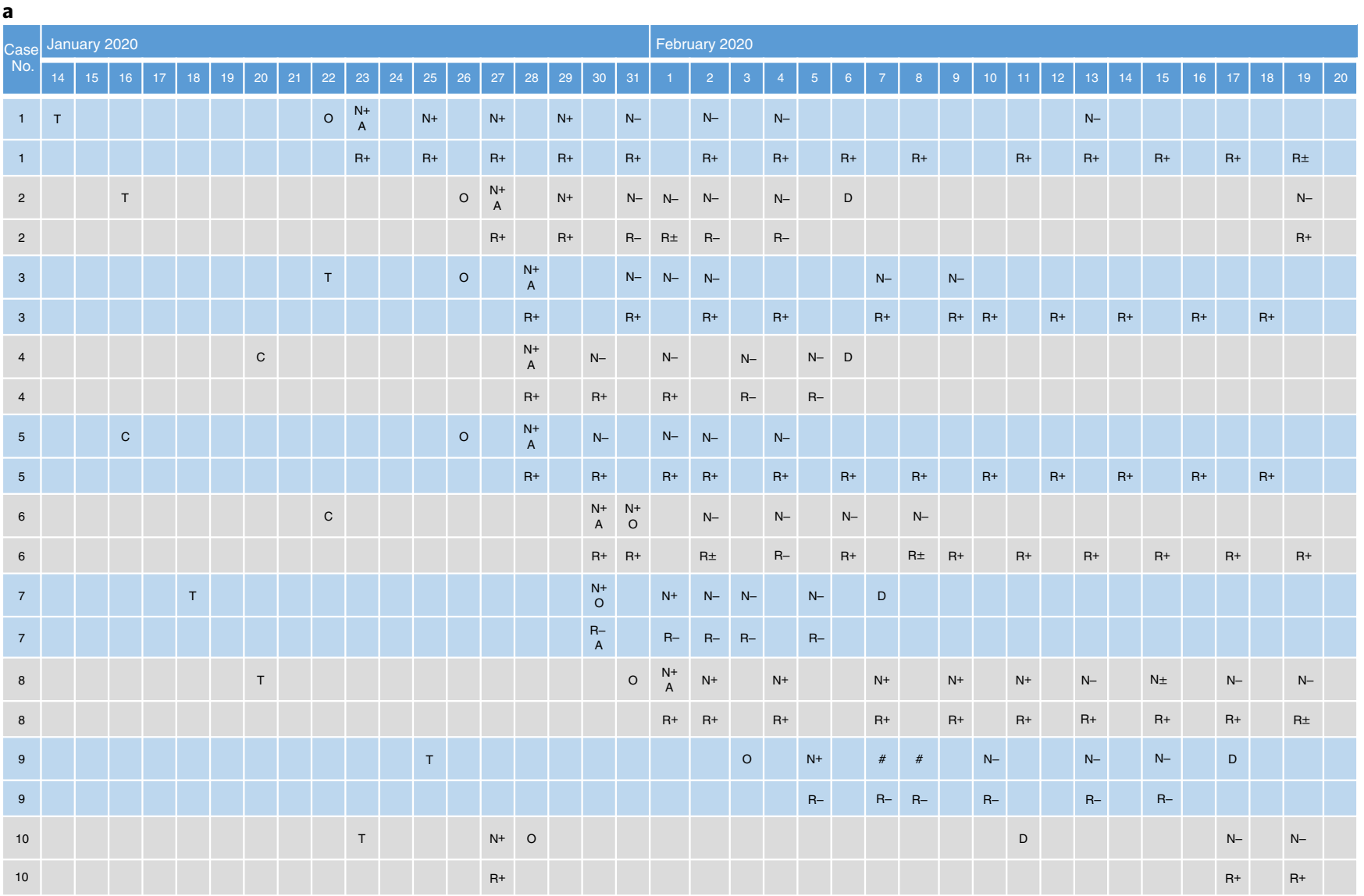

$\mathrm{T}$, travel to epidemic area; $\mathrm{C}$, contact with confirmed cases; $\mathrm{O}$, onset of symptom; $\mathrm{A}$, admission; $\mathrm{N}+$, nasopharyngeal swab positive; $\mathrm{N}-$, nasopharyngeal swab negative; $\mathrm{N}_{ \pm}$, nasopharyngeal swab weak positive; R+, rectal swab positive; R土, rectal swab weak positive; R-, rectal swab positive; $\mathrm{D}$, date of discharge; \#, failed samples
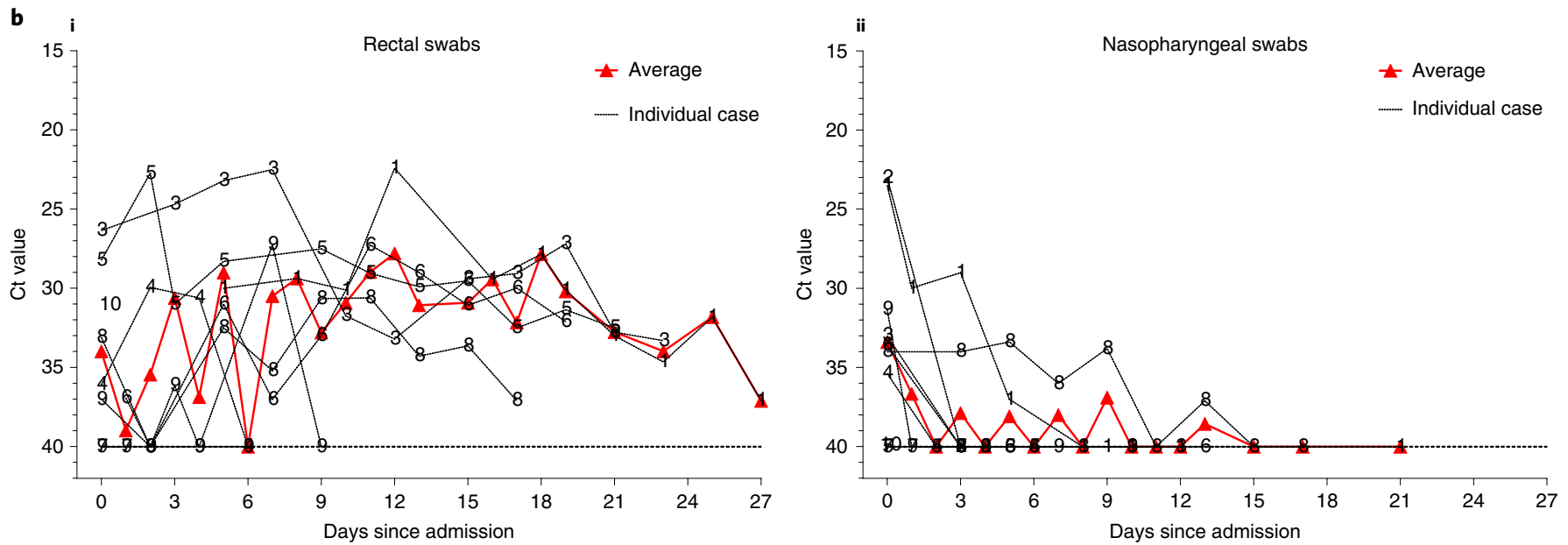

Fig. 1 | Chronology of major epidemiological events and molecular testing results of $\boldsymbol{n}=10$ independent pediatric patients confirmed with SARS-CoV-2 infection. a, Dates of exposure, illness onset and sampling and real-time RT-PCR results of nasopharyngeal swabs and rectal swabs. The total number of patients was $n=10$ and real-time RT-PCR was assayed only once for one type of sample at one time point from one independent patient. Colors in the figure represent individual patients. b. Chronological changes in Ct values of Orf1ab and $\mathrm{N}$ genes using real-time RT-PCR after hospital admission. The Ct values of Orflab and $N$ genes on real-time RT-PCR detected in rectal swabs obtained from $n=10$ independent patients (i) and Ct values in nasopharyngeal swabs from $n=10$ independent cases (ii). The $C t$ value is supposed to be inversely related to viral RNA copy numbers and a value of 40 means the virus is molecularly undetectable.

sion), seven showed elevated interleukin (IL)-17F and five of these patients had concurrent elevation of IL-22 (Supplementary Table 1). Five patients had elevated IL-6.

All patients received antiviral therapy with $\alpha$-interferon oral spray initiated from admission $(8,000 \mathrm{U}$, two sprays, three times a day). Patient 1 was the very first pediatric case of SARS-CoV-2 infection diagnosed in Guangzhou and also treated with azithromycin $10 \mathrm{mg} \mathrm{kg}^{-1}$ per day for $5 \mathrm{~d}$ and IVIG $300 \mathrm{mg} \mathrm{kg}^{-1}$ per day for $3 \mathrm{~d}$. No patient required respiratory support or intensive care unit care. 
We followed the pattern of viral excretion from respiratory and gastrointestinal tracts in all ten patients by a chronological series of nasopharyngeal and rectal swab samples using real-time RT-PCR (Fig. 1a). Patient 4 was asymptomatic but tested positive on multiple occasions. Patient 6 was asymptomatic on the day his nasopharyngeal swab tested positive and then developed nasal congestion and rhinorrhea the following day. The remaining eight patients had positive tests soon after the onset of symptoms. In addition, eight of ten patients also had real-time RT-PCR-positive rectal swabs, suggesting potential fecal viral excretion. Moreover, eight of ten patients (patients 1-6, 8 and 10) demonstrated persistently positive real-time RT-PCR tests of rectal swabs after their nasopharyngeal testing had become negative.

Patients 2, 4, 7 and 10 were discharged home after two consecutive negative real-time RT-PCR tests (separated by at least $24 \mathrm{~h}$ ) on rectal swabs. Their nasopharyngeal and rectal swabs were repeated weekly after discharge. Patients 4 and 7 remained negative during follow-up but patient 2 had a positive rectal swab again $13 \mathrm{~d}$ after discharge. Interestingly, the mother of patient 2, who was hospitalized for SARS-CoV-2 infection (COVID-19) and discharged from a different hospital during the same time period, also had a positive rectal swab test on the same day as her child. A similar phenomenon was also observed in patient 10 . He was hospitalized in the Eighth People's Hospital of Guangzhou between 27 January and 11 February and discharged after two consecutive negative results for both nasopharyngeal and rectal swabs obtained $24 \mathrm{~h}$ apart. $\mathrm{He}$ was however re-admitted to our hospital because his rectal swab test became positive again on 17 February, although his nasopharyngeal swab test remained negative. As of $20 \mathrm{Feb}$, all remaining patients (patients 1, 3, 5, 6, 8 and 9) were still testing positive for rectal swabs and continued to be under hospital isolation and observation, although they had all recovered from their illness and had become asymptomatic.

As suggested in a recent study on a similar topic ${ }^{7}$, we used the cycle threshold $(\mathrm{Ct})$ values of the serial rectal and nasopharyngeal swab tests to approximately indicate viral load (inversely related to $\mathrm{Ct}$ value) in these patients to show its change over time. Viral RNA measurements suggest that viral shedding from the digestive system might be greater and last longer than that from the respiratory tract (Fig. 1b).

Clinical features of SARS-CoV-2 infection in adults have been reported elswhere ${ }^{4,8,9}$. However, few pediatric cases are published and their clinical features have yet to be documented. Compared to adult patients, the ten pediatric patients reported here had clinically milder symptoms and showed fewer alterations in radiological and laboratory testing parameters. For example, none of the ten patients showed clear clinical signs or chest X-ray findings consistent with pneumonia, a typical feature seen in the initial adult patients ${ }^{4,6}$. Mild and atypical presentations of the infection in children may make it difficult to detect. Indeed, all the patients reported here were found through screening of suspected cases.

We also observed positive real-time RT-PCR results in rectal swabs in eight out of ten pediatric patients, which remained detectable well after nasopharyngeal swabs turned negative, suggesting that the gastrointestinal tract may shed virus and fecal-oral transmission may be possible. Indeed, fecal-oral transmission does exist with other respiratory viruses ${ }^{10}$. These findings also suggest that rectal swab-testing may be more useful than nasopharyngeal swab-testing in judging the effectiveness of treatment and determining the timing of termination of quarantine ${ }^{12}$. However, we do not have evidence of replication-competent virus in fecal swabs, which is required to confirm the potential for fecal-oral transmission.

\section{Online content}

Any methods, additional references, Nature Research reporting summaries, source data, extended data, supplementary information, acknowledgements, peer review information; details of author contributions and competing interests; and statements of data and code availability are available at https://doi.org/10.1038/s41591020-0817-4.

Received: 10 February 2020; Accepted: 2 March 2020; Published online: 13 March 2020

\section{References}

1. Wang, C., Horby, P. W., Hayden, F. G. \& Gao, G. F. A novel coronavirus outbreak of global health concern. Lancet 395, 470-473 (2020).

2. Munster, V. J., Koopmans, M., van Doremalen, N., van Riel, D. \& de Wit, E. A novel coronavirus emerging in China-key questions for impact assessment. N. Engl. J. Med. 382, 692-694 (2020).

3. Coronavirus Disease (COVID-2019) Situation Report 36 (WHO, 2020).

4. Chen, N. et al. Epidemiological and clinical characteristics of 99 cases of 2019 novel coronavirus pneumonia in Wuhan, China: a descriptive study. Lancet 395, 507-513 (2020)

5. Huang, C. et al. Clinical features of patients infected with 2019 novel coronavirus in Wuhan, China. Lancet 395, 497-506 (2020).

6. Wang, D. et al. Clinical characteristics of 138 hospitalized patients with 2019 novel coronavirus-infected pneumonia in Wuhan, China. JAMA https://doi. org/10.1001/jama.2020.1585 (2020).

7. Zhu, N. et al. A novel coronavirus from patients with pneumonia in China, 2019. N. Engl. J. Med. 382, 727-733 (2020).

8. Holshue, M. L. et al. First case of 2019 novel coronavirus in the United States. N. Engl. J. Med. 382, 929-936 (2020).

9. Guan, W. et al. Clinical characteristics of 2019 novel coronavirus infection in China. N. Engl. J. Med. https://doi.org/10.1056/NEJMoa2002032 (2020).

10. Zhu, Z. et al. Extra-pulmonary viral shedding in H7N9 Avian Influenza patients. J. Clin. Virol. 69, 30-32 (2015).

Publisher's note Springer Nature remains neutral with regard to jurisdictional claims in published maps and institutional affiliations.

(c) The Author(s), under exclusive licence to Springer Nature America, Inc. 2020 


\section{Methods}

Study design and participants. In this single-center prospective observational study, between 22 January 2020 and 20 February 2020, 745 'highly suspected' children were screened by real-time RT-PCR using nasopharyngeal swabs to detect people with SARS-CoV-2 infection. Ten children tested positive and were admitted to Guangzhou Women and Children's Medical Center. They were all recruited to this study. A child who came in contact with a patient confirmed with SARS-CoV-2 infection within the previous $14 \mathrm{~d}$ or who was a member of a familial outbreak of the virus was considered 'highly suspected'. All 'highly suspected' children were placed under home quarantine until a SARS-CoV-2 diagnosis was ruled out by real-time RT-PCR.

Data on demographics, contact/exposure history, clinical presentations on admission, chest X-ray and laboratory tests were collected. Laboratory data collected on each patient included complete blood count, urine and stool analysis, blood biochemistry, coagulation function, biomarkers of infection, viral testing through nasopharyngeal and rectal swabs, as well as immunological testing (six items) and a cytokine profile (14 cytokines). Examination of viral excretion from the respiratory and gastrointestinal tract was conducted by serial real-time RTPCR of nasopharyngeal and rectal swab samples.

Guangzhou Women and Children's Medical Center, located in Guangzhou, Guangdong Province, China, is a specialized tertiary pediatric hospital caring for children under 18 years of age. To effectively manage patients with SARS-CoV-2 infection, the Municipal Government has put in place a centralized care policy and this hospital is a designated treatment center for children with SARS-CoV-2 infection in Guangdong Province, of which Guangzhou is the capital city. All highly suspected and confirmed pediatric cases in Guangzhou were admitted to this hospital after the SARS-CoV-2 infection was classified as a reportable disease in the Infectious Disease Law and Health and Quarantine Law in China on 20 January 2020. Diagnosis of SARS-CoV-2 infection was made using real-time RT-PCR in the virology laboratory of our hospital and then further confirmed by either Guangzhou Center for Disease Control and Prevention (CDC) or Guangdong Provincial CDC. Nasopharyngeal and rectal swabs were collected upon admission and then every $1-3 \mathrm{~d}$ throughout the hospitalization period. SARS-CoV-2 was tested through real-time RT-PCR of 2019-nCoVRNA as previously reported ${ }^{6}$. Two target genes, including open reading framelab (ORF1ab) and nucleocapsid protein $(N)$, were simultaneously amplified and tested during the real-time RT-PCR assay.

Target 1 (ORF1ab):

Forward primer CCCTGTGGGTTTTACACTTAA;

Reverse primer ACGATTGTGCATCAGCTGA;

The probe 5'-VIC-CCGTCTGCGGTATGTGGAAAGGTTATGG-BHQ1-3'

Target $2(N)$ :

Forward primer GGGGAACTTCTCCTGCTAGAAT;

Reverse primer CAGACATTTTGCTCTCAAGCTG;

The probe 5' -FAM- TTGCTGCTGCTTGACAGATT-TAMRA-3'.

A Ct value $<37$ was defined as positive and $>40$ was defined as negative. These diagnostic criteria were based on the recommendation by the National CDC (China) (http://ivdc.chinacdc.cn/kyjz/202001/t20200121_211337.html). A medium load, defined as a Ct value of 37 to 40 , required confirmation by at least two repeats.

In addition, other potential respiratory viruses were also tested using real-time RT-PCR approved by the China Food and Drug Administration. These included influenza-A virus (H1N1, H3N2, H7N9), influenza B virus, respiratory syncytial virus, parainfluenza virus, adenovirus, SARS coronavirus (SARS-CoV) and MERS coronavirus (MERS-CoV).

Data collection. Microsoft Excel (MS Excel 2013, v.15.0) was used for data collection of the epidemiological and clinical information. Epidemiological data were obtained through standard epidemiological questionnaire and interviews ${ }^{5}$. Clinical information, including clinical presentations and management, were obtained by reviewing clinical charts, nursing records and results of laboratory testing, chest X-rays (Canon SM-50HF-B-D-C mobile DR: tube voltage $65 \mathrm{kV}$, tube current $125 \mathrm{~mA}$, exposure time $0.04 \mathrm{~s}$ ) and CT scans (Toshiba's Aquilion 64-slice CT: tube voltage $120 \mathrm{kV}$, tube current $50-100 \mathrm{~mA}$, tube rotation time $0.5 \mathrm{~s} \mathrm{r}^{-1}$, slice thickness $1.0 \mathrm{~mm}$, slice interval $1.0 \mathrm{~mm}$ ) for all children with laboratory-confirmed 2019-nCOV infection. Clinical outcome data were recorded up to the date of drafting this manuscript on 20 February 2020. The accuracy of clinical data was confirmed by two pediatricians caring for the patients.
To assess whether SARS-CoV-2 infection could induce an intense immune response that would result in cytokine storms, we collected peripheral blood on admission and tested six immunological indexes and 14 cytokines in these patients. Plasma cytokines (interferon- $\gamma$, IL-1 $\beta$, IL-2, IL-4, IL-5, IL-6, IL-8, IL-10, IL-12p70, IL-17A, IL-17F, IL-22, tumor necrosis factor- $\alpha$ and tumor necrosis factor- $\beta$ ) were measured by using Human Cytokine Standard 27-Plex Assays panel (HumanTh1/ Th2/Th1718-plex, Tianjin Quantobio) and FACSCanto II Flow Cytometry system (Becton Dickinson) for all patients according to the manufacturer's instructions. The plasma samples from four healthy adults were used as controls for cross comparison.

Statistical analysis. Characteristics of the patients were described and summarized. These included epidemiological data, demographics, clinical signs and symptoms on admission, laboratory results, co-infection with other respiratory pathogens, chest radiographic findings, treatment received for 2019-nCOV and clinical outcomes. Ct values of Orflab and N gene using real-time RT-PCR assay were inversely related to viral RNA copy number, with Ct values of 32.04, 28.81, 25.14 and 21.54 corresponding to $5.27 \times 10^{4}, 5.27 \times 10^{5}, 5.27 \times 10^{6}$ and $5.27 \times 10^{7}$ copies per ml. Thus, Ct values were used to approximately evaluate the viral load detected in nasopharyngeal and rectal swabs. A chi-squared test was performed for between-group comparisons of percentages. All statistical tests were two-sided with $P<0.05$ considered as statistically significant and were performed using $\mathrm{R}$ (v.3.1.2; R Foundation for Statistical Computing).

Ethics approval. Ethics approval was obtained from the Ethics Committee of Guangzhou Women and Children's Medical Center and written informed consents were obtained from the parents of the included children. Data collection and analysis were also required by the National Health Commission of the People's Republic of China to be part of a continuing public health outbreak investigation.

Reporting Summary. Further information on research design is available in the Nature Research Reporting Summary linked to this article.

\section{Data availability}

The data in this study have been shared with the World Health Organization and will be available upon request and approval by a data access committee. The data access committee comprises four corresponding authors and there is no restriction to data access.

\section{Acknowledgements}

This work was funded by the National Key Research and Development Program of China (2019YFB1404803) and Guangzhou Regenerative Medicine Guangdong Laboratory (2020GZR110306001). We thank the CDCs of Guangzhou City and Guangdong Province for helping with confirmation of the diagnosis of the viral infection.

\section{Author contributions}

J.T., H.X. and S.G. contributed to the study design and data interpretation. H.L., J.T., K.Z. and Y.X. contributed to data analysis, data interpretation and writing of the manuscript. Y.X., X.L., B.Z., C.F., Y.G., Q.G., X.S., D.Z., H.S.L. and J.S. contributed to clinical management, patient recruitment and data collection. H.Z. and B.Z. contributed to critical revision of the manuscript. All authors reviewed and approved the final version of the manuscript.

\section{Competing interests}

The authors declare no competing interests.

\section{Additional information}

Extended data is available for this paper at https://doi.org/10.1038/s41591-020-0817-4. Supplementary information is available for this paper at https://doi.org/10.1038/ s41591-020-0817-4.

Correspondence and requests for materials should be addressed to H.X., J.T., K.Z. or S.G.

Peer review information Alison Farrell was the primary editor on this article and managed its editorial process and peer review in collaboration with João Monteiro and the rest of the editorial team.

Reprints and permissions information is available at www.nature.com/reprints. 

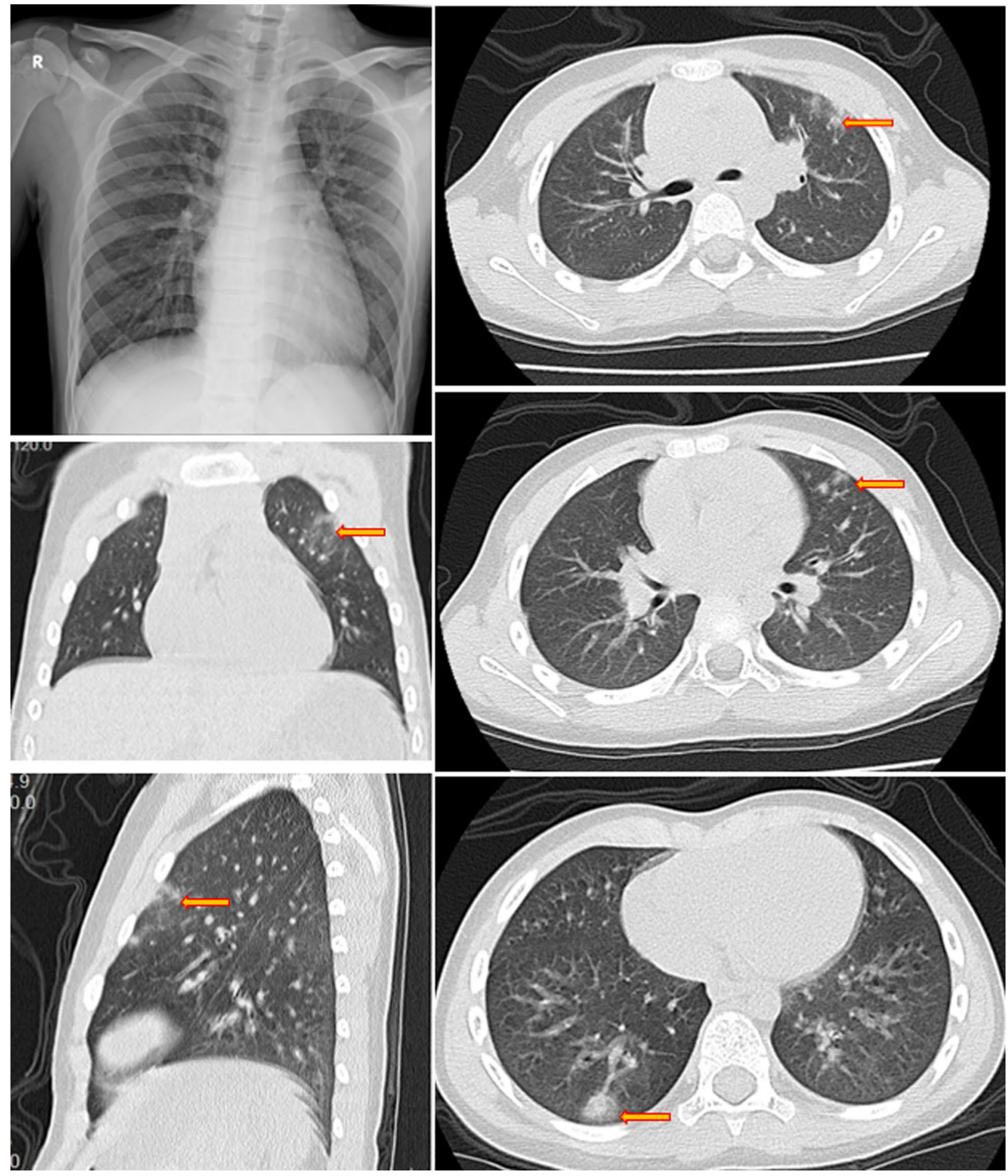

Extended Data Fig. 1| An example of chest x-ray and computed tomography (CT) scan images at hospital admission (case4). An example of chest x-ray and computed tomography (CT) scan images at hospital admission (case4). 


\section{natureresearch}

\section{Reporting Summary}

Nature Research wishes to improve the reproducibility of the work that we publish. This form provides structure for consistency and transparency in reporting. For further information on Nature Research policies, see Authors \& Referees and the Editorial Policy Checklist.

\section{Statistics}

For all statistical analyses, confirm that the following items are present in the figure legend, table legend, main text, or Methods section.

n/a Confirmed

$\square$ \. The exact sample size $(n)$ for each experimental group/condition, given as a discrete number and unit of measurement

$\square \bigotimes$ A statement on whether measurements were taken from distinct samples or whether the same sample was measured repeatedly

The statistical test(s) used AND whether they are one- or two-sided

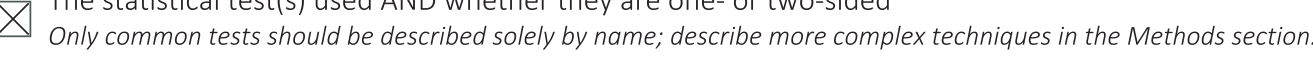

\ $\square$ A description of all covariates tested

$\bigotimes \square$ A description of any assumptions or corrections, such as tests of normality and adjustment for multiple comparisons

$\triangle$ A full description of the statistical parameters including central tendency (e.g. means) or other basic estimates (e.g. regression coefficient)

AND variation (e.g. standard deviation) or associated estimates of uncertainty (e.g. confidence intervals)

$\varnothing$ For null hypothesis testing, the test statistic (e.g. $F, t, r$ ) with confidence intervals, effect sizes, degrees of freedom and $P$ value noted

Give $P$ values as exact values whenever suitable.

Х $\square$ For Bayesian analysis, information on the choice of priors and Markov chain Monte Carlo settings

Х $\square$ For hierarchical and complex designs, identification of the appropriate level for tests and full reporting of outcomes

$\bigotimes \square$ Estimates of effect sizes (e.g. Cohen's $d$, Pearson's $r$ ), indicating how they were calculated

Our web collection on statistics for biologists contains articles on many of the points above.

\section{Software and code}

Policy information about availability of computer code

Data collection Ms Excel 2013, version 15.0

Data analysis $\quad$ R (version 3.1.2; The R Foundation for Statistical Computing, Vienna, Austria)

For manuscripts utilizing custom algorithms or software that are central to the research but not yet described in published literature, software must be made available to editors/reviewers. We strongly encourage code deposition in a community repository (e.g. GitHub). See the Nature Research guidelines for submitting code \& software for further information.

\section{Data}

Policy information about availability of data

All manuscripts must include a data availability statement. This statement should provide the following information, where applicable:

- Accession codes, unique identifiers, or web links for publicly available datasets

- A list of figures that have associated raw data

- A description of any restrictions on data availability

The data in this study has been shared with the WHO and will be available upon request and approval by a data access committee.

\section{Field-specific reporting}

Please select the one below that is the best fit for your research. If you are not sure, read the appropriate sections before making your selection.
$\bigotimes$ Life sciences
Behavioural \& social sciences
Ecological, evolutionary \& environmental sciences

For a reference copy of the document with all sections, see nature.com/documents/nr-reporting-summary-flat.pdf 


\section{Life sciences study design}

All studies must disclose on these points even when the disclosure is negative.
Sample size
Single-center prospective observational study. Included a total of 10 cases.

Data exclusions

No data were excluded.

Replication

For medium viral load, defined as a Ct-value of 37 to 40, required confirmation by at least 2 replications.

Randomization

Prospective observational study, no randomization.

Blinding

Prospective observational study, no blinding.

\section{Reporting for specific materials, systems and methods}

We require information from authors about some types of materials, experimental systems and methods used in many studies. Here, indicate whether each material, system or method listed is relevant to your study. If you are not sure if a list item applies to your research, read the appropriate section before selecting a response.

\begin{tabular}{l|l} 
Materials \& experimental syst \\
\hline$n / a$ & Involved in the study \\
\hline & $\square$ Antibodies \\
$\square$ & $\square$ Eukaryotic cell lines \\
$\square$ & $\square$ Animals and other organisms \\
$\square$ & $\bigotimes$ Human research participants \\
$\square$ & $\bigotimes$ Clinical data
\end{tabular}

\begin{tabular}{l|l}
\multicolumn{2}{l}{ Methods } \\
\hline n/a & Involved in the study \\
\hline & $\square$ ChIP-seq \\
$\square$ & $\square$ Flow cytometry \\
$\square$ MRI-based neuroimaging
\end{tabular}

\section{Human research participants}

Policy information about studies involving human research participants

Population characteristics

A total of 10 pediatric SARS CoV-2 infection cases, 6 were males and 4 were females, with age ranging from 2 months to 15 years. All patients received antiviral therapy with $\alpha$-interferon oral spray initiated from the admission $(8000 \mathrm{U}, 2$ sprays, thrice a day). No children required respiratory support or ICU care.

Recruitment

Since the research constitutes an analysis of existing data of pediatric SARS CoV-2 infection cases, there was no specific recruitment process involved.

Ethics oversight

We obtained approval by the ethics committee of Guangzhou Women and Children's Medical Center and written informed consents were obtained from the parents of the included patients before enrollment.

Note that full information on the approval of the study protocol must also be provided in the manuscript.

\section{Clinical data}

Policy information about clinical studies

All manuscripts should comply with the ICMJE guidelines for publication of clinical research and a completed CONSORT checklist must be included with all submissions.

Clinical trial registration

Study protocol

Data collection

Outcomes
This is not a clinical trail.

Characteristics of pediatric SARS CoV-2 infection and potential evidence for persistent fecal viral shedding. Besides this description paper, no other protocol are available.

Data was acquired at the Guangzhou Women and Children's Medical Center, located in Guangzhou, Guangdong province, China We recruited all children with confirmed 2019-nCoV infection who were admitted to Guangzhou Women and Children's Medical Center between Jan.22-Feb.22, 2020.

Epidemiological characteristics, clinical characteristics, and Oasopharyngeal and rectal swabs SARS CoV-2 testing results using real-time RT-PCR 


\section{Plots}

Confirm that:

Х The axis labels state the marker and fluorochrome used (e.g. CD4-FITC).

Х The axis scales are clearly visible. Include numbers along axes only for bottom left plot of group (a 'group' is an analysis of identical markers).

Х All plots are contour plots with outliers or pseudocolor plots.

$\bigotimes$ A numerical value for number of cells or percentage (with statistics) is provided.

\section{Methodology}

Sample preparation

Instrument

Software

Cell population abundance

Gating strategy
Take the serum after centrifugation, test on the machine or test after thawing in - $80^{\circ}$ low temperature storage, avoid repeated freezing and thawing.

BD FACSCanto II

BD FACSDiVa,FCAP Array 3.0

The fluorescent intensity of PE on the beads is quantified on a flow cytometer. Concentrations of a protein of interest in the samples can be obtained by comparing the fluorescent signals to those of a standard curve generated from a serial dilution of a known concentration of the analyte.

Adjust FSC and SSC so that the microsphere community is within the predetermined range. The smaller microsphere group is set as "Gate S4", and the larger microsphere group is set as "Gate S5".

Х Tick this box to confirm that a figure exemplifying the gating strategy is provided in the Supplementary Information. 